\title{
Editorial
}

\section{Costing a healthy diet: measurement and policy implications}

Health-related food taxes and subsidies are a hot topic in public health research. A number of countries have recently introduced such pricing strategies with the aim to promote healthier food choices. One of the most recent examples is Mexico, which introduced an excise tax on sugar-sweetened beverages of 1 peso per litre (approximately a $10 \%$ price increase based on 2013 prices) and a sales tax on several highly energy-dense foods (containing $1151 \mathrm{~kJ}(\geq 275 \mathrm{kcal})$ per $100 \mathrm{~g})^{(1)}$.

The relatively high cost of healthy food is often cited as an important argument to introduce food taxes and subsidies and thereby make healthy food (relatively) more affordable ${ }^{(2)}$. Likewise, the cost of healthy foods is listed by consumers, and particular lower socio-economic groups, as an important barrier to purchasing healthier foods ${ }^{(3)}$. However, there is much debate in the literature about whether healthy foods indeed cost more, where it has been argued that it is possible to eat healthily on a limited budget, but also that this is only possible if people are willing to divert hugely from culturally acceptable dietary patterns ${ }^{(4)}$. A recent systematic review and metaanalysis revealed that, on average, healthier diets cost more than unhealthy diets ${ }^{(5)}$; however, that review also highlights the importance of carefully considering the metric of healthfulness, intensity of contrast and unit of comparison $^{(5)}$. As it turns out, measuring the price of foods and diets is a lot more complicated than it might appear at first sight.

The current issue of Public Health Nutrition features a new systematic review that looked into measuring food prices in more detail ${ }^{(6)}$. The aim of that review was to determine similarities and differences in metrics and results between tools, protocols and methods used for monitoring (Australian) healthy food prices and affordability. The authors reviewed thirty-nine reports and twenty-four journal articles which described fifty-nine distinct food pricing surveys undertaken in Australia. The included surveys measured the cost of healthy foods by using a food basket approach. This approach involves measuring the cost of a predetermined selection (basket) of foods by collecting prices of these foods from retail outlets (mostly supermarkets). From these surveys, the authors identified six 'major' food pricing tools (used in multiple areas and multiple time periods) and five 'minor' food pricing tools (only used in one survey or during one time period).

The authors identified large differences in the tools and protocols used in the different surveys, even when the same food pricing tool was used in different areas or time periods. They observed differences in: selection of 'healthy' basket contents; reference household composition; inclusion of availability and/or quality measures; household income sources; store sampling methods; season of data collection; and data collection protocols and analysis. This divergence in measurements makes it virtually impossible to make food price comparisons between countries or regions or over time and the authors argue it is important to establish a single measurement of (healthy) food costs. The INFORMAS (International Network for Food and Obesity/non-communicable diseases Research, Monitoring and Action Support) network is currently leading initiatives for establishing such a standardized monitoring approach ${ }^{(7)}$.

\section{A typical (healthy/unhealthy) diet}

A remarkable finding from the systematic review was that only one of the reviewed surveys measured the cost of a typical (unhealthy) diet to allow comparison between the cost of a healthy diet and the current diet. Recently, standardized methods to assess the relative price difference between a healthy and current diet have been piloted in Australia ${ }^{(8)}$. The results of that pilot suggested that healthy diets can be more affordable than the current Australian diet, particularly because people currently spend a relatively large proportion of their household budget on alcohol and discretionary foods (a family of two adults and two children was found to spend over $58 \%$ of its food dollars on discretionary choices). Nevertheless, the individual prices of some healthy foods, particularly meat, dairy and vegetables, were found to be relatively expensive compared with energy-dense discretionary foods ${ }^{(8)}$. Also, the study found that while a diet consistent with dietary guidelines was affordable for families on a median income ( $18 \%$ of disposable income), it was less affordable for low-income families ( $28 \%$ of their household disposable income) ${ }^{(8)}$.

Another remarkable finding from the systematic review featured in this issue ${ }^{(6)}$ was that ten of eleven identified 'healthy basket' food pricing tools did not fully align with the principles of the most recent Australian Dietary Guidelines. This is because studies added discretionary foods and/or commonly consumed unhealthy foods to the 'healthy' basket apparently to 'adjust the energy content of the basket'. The authors do not discuss this topic in depth, other than saying that most healthy baskets do not constitute a diet consistent with dietary guidelines; however, 
this is a very remarkable observation. Adding energydense foods (e.g. sugar, oil, processed meat, snacks) to healthy food baskets to obtain the required energy levels seems highly controversial. It makes it look as if, by following the dietary guidelines, it is impossible to consume the required energy amount (say, $8368 \mathrm{~kJ} / \mathrm{d}$ $(2000 \mathrm{kcal} / \mathrm{d}))$. This is odd, as one could argue the opposite is true. Meeting all daily micro- and macronutrient requirements while ensuring a good energy balance is relatively difficult and leaves little room for discretionary foods (i.e. you need all $8368 \mathrm{~kJ}$ (2000 kcal) to get the required nutrients from healthy foods). Most people consume more kilojoules than they need without meeting recommended intakes for a number of nutrients. Therefore, people should choose foods that are high in nutrients but low to moderate in energy content; that is, meeting nutrient recommendations must go hand in hand with keeping kilojoules under control $^{(9)}$. Another reason for adding discretionary foods to healthy food baskets could be to make the basket more realistic and in line with what people are currently eating. While this makes sense, it would be better to have a separate basket containing foods from the current diet and compare this with the healthy diet that fully aligns with dietary guidelines. Authors could also opt to add some sensitivity analyses (e.g. have varying baskets meeting $50-100 \%$ of dietary guidelines).

\section{Price metrics}

Another gnawing issue in measuring food costs is the metrics used for food price. There are three different potential metrics, often leading to very different results: price per weight $(\$ / g)$; price per serving (\$/serving); and price per energy unit $(\$ / \mathrm{kJ}$ or $\mathrm{kcal})$. A recent systematic review and meta-analysis revealed that the most striking example for difference in findings between the used metrics can be observed in dairy products, where healthier options were found to be \$US 0.004 less expensive per serving but \$US 0.21 more expensive per $837 \mathrm{~kJ}$ $(200 \mathrm{kcal})^{(5)}$. The authors clarify that this divergence in findings can be explained by the fact that whole milk contains nearly twice the energy as fat-free milk, meaning that nearly double the amount of fat-free milk must be purchased to achieve equivalent energy. As explained in their paper, these findings highlight the dangers of socalled 'circular reasoning' (e.g. selecting a metric based on energy content and then evaluating price differences per unit of energy) and the importance of identifying the most accurate unit of comparison for any individual or public health decision about price differences of foods ${ }^{(5)}$.

The issue of price metrics has long been a hot topic of debate in the literature. In the early 2000s, Drewnowski, Darmon and others started publishing a series of papers examining the issue of energy density and energy cost,

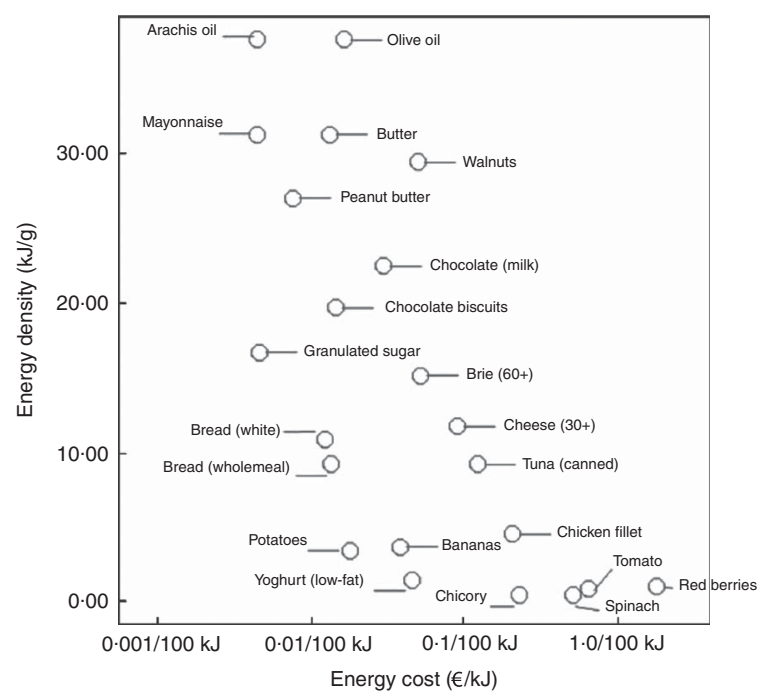

Fig. 1 Relationship between energy density and energy cost (from Waterlander et al. ${ }^{(46)}$ )

looking either at individual foods ${ }^{(10,11)}$ or dietary patterns ${ }^{(12-14)}$. These studies produced the well-known diagrams as displayed in Fig. 1, showing that foods high in energy density (e.g. fats, sugar) are relatively cheaper per kilojoule than foods with a low energy density (e.g. fruits, vegetables). In 2009, this finding was challenged by Lipsky, who argued that this result is merely a mathematical artefact (or circular reasoning as explained above) ${ }^{(15)}$. To prove the point, Lipsky used a random-number generator to create numbers for three variables: energy (A), grams (B) and total price $(\mathrm{C})$, and then used these variables to create A/B (energy density) and C/A (energy cost). Also, she measured actual food price data from US supermarkets. Subsequently, she drew scatterplots using the random data and observational data and compared the two. The author found that the same relationship was found in the randomly generated data and the genuinely observed data, meaning that the 'energy density/energy cost' observation is caused primarily by having energy in both the independent and dependent variable ${ }^{(15)}$.

As explained above, using energy in both the numerator and denominator causes problems. On top of that, it can be argued that people don't buy food per kilojoule, providing a second reason why this metric is invalid. While we appreciate these problems, we would like to highlight that this doesn't mean that energy cost is not a valid measure at all. Arguably, when people buy food they do inherently think of energy density one way or the other. For example, if you are hungry and have \$US 3 to spend, would you buy a bag of apples or a Big Mac? While this example shows that \$US 3 might provide you both a healthy and unhealthy option (showing that you can buy healthily for little money), apples are not filling you up the same way as a Big Mac does. This importance was also highlighted by Darmon and Maillot in their response to 
Lipsky's criticism: 'price is a well-known determinant of food choice and because the need for energy is probably the only nutritional requirement specifically and acutely perceived by individuals, we continue to believe that energy cost may influence dietary behaviour ${ }^{(16)}$.

To date, there is no consensus in the literature on what the best price metric is. However, there are a number of papers that argue (using price per gram or per serving) you can eat healthily on a limited budget ${ }^{(17,18)}$. Using the cost of the diet, some observational studies found that certain population groups can achieve higher-quality diets at a lower cost than other groups ${ }^{(19,20)}$. Others report that if foods are carefully selected it is possible to consume a low-cost diet relatively low in energy density and high in nutrient density ${ }^{(21-23)}$ but this requires a motivated and knowledgeable shopper ${ }^{(18)}$. While, strictly speaking, it might be true that people can eat healthily on a limited budget, we have to be careful in interpreting this finding correctly. The fact that people can, in theory, buy healthy food for little money does not mean that the price of food is not a barrier for healthy eating. First, it is still quite hard to eat a healthy and varied diet on a limited budget; you can't expect people to eat lentils every day. But, more importantly, it is a lot easier to eat unhealthily on a limited budget. Arguably, if people are restrained by other factors (e.g. job stress, financial stress, etc.), consuming a healthy diet might not be on top of their priority list. Even when healthy and unhealthy foods are the same price at point of purchase, if it's easier and more convenient to choose unhealthy foods (think of all the unhealthy foods you can buy for a few dollars), healthy foods will still not be the choice of preference.

\section{Cost of convenience}

Not only the absolute cost, but also the perceived inconvenience is a major barrier to healthy food choices when choosing meals ${ }^{(24,25)}$. Households perceive a lack of time for food preparation and food manufacturers use this sentiment to develop and promote convenience foods ${ }^{(26,27)}$. Indeed, global food manufacturers have a vested interest in the production and sale of ultraprocessed foods because production costs are low and highly processed foods have a long shelf-life and a high retail value ${ }^{(28,29)}$. Unfortunately, there is a clear trade-off between nutritional quality and convenience, where consuming meals prepared with wholesome foods is associated with good health and low risk of disease while consumption of ultra-processed energy-dense foods is associated with increased risk of obesity and noncommunicable diseases ${ }^{(30,31)}$.

Meal preparation is influenced by the cost of purchasing food and the cost of time as well as taste, culture and other influences. For some people, the provision of homecooked meals provides benefits such as enjoyment of cooking, social interaction, relaxing, a nurturing role and the opportunity cost of time for other activities. For others cooking is considered a chore, with little time available or prioritized for cooking ${ }^{(32)}$. Time is not usually factored into the price of food preparation but can be a barrier to preparing meals ${ }^{(33)}$. The evidence on how to incorporate time is limited ${ }^{(33)}$, with very few studies in the literature incorporating external costs. An American study evaluated the cost of various pre-prepared and home-made ingredients (e.g. apple sauce) and meals (e.g. lasagne). The time to prepare the items was calculated and the hourly wage of a food preparer was used as the cost of time. When the cost of time was included, the processed items cost less than the home recipe for all items, particularly grains, vegetables and fruit ${ }^{(34)}$. The cost to prepare the US Department of Agriculture's Thrifty Food Plan was met by $62 \%$ of low-income households, but when the time costs were included only $13 \%$ could purchase the required foods ${ }^{(33)}$.

\section{Conclusion}

The systematic review featured in this issue of Public Health Nutrition ${ }^{(6)}$ and other literature show that measuring the cost of food both accurately and meaningfully is very challenging. Nevertheless, it is clear that the cost of food is a major issue in public health nutrition and should therefore be seriously considered for policy intervention. There is growing evidence suggesting that health-related food taxes and subsidies (e.g. a soft drink tax or a fruit and vegetable subsidy) are effective in improving population diets $^{(35-40)}$. Likewise, there is evidence that fiscal policy might not only work to improve the health profile of population diets, but also improve the environmental footprint $^{(41)}$, showing the important link between agriculture, health and global greenhouse gas emissions ${ }^{(42)}$.

Health-related food taxes or subsidies are, however, not the only mechanism to alter food prices, where we should not forget that certain existing policies heavily influence the price of food. For example, the European Common Agricultural Policy (CAP) currently heavily subsidizes beef and milk production which leads to an oversupply of cheap meat in the market. Similarly, CAP keeps fruit and vegetable prices high by implementing price guarantees, product withdrawal and import tariffs for fruit and vegetables from outside the $\mathrm{EU}^{(43)}$. The history of subsidies both direct and indirect - has created an agricultural system focused on creating cheap beef and other animalsource foods in most developing nations ${ }^{(44)}$. This is problematic as a recent American study found that, among US adults, higher consumption of energy from subsidized food commodities was associated with a greater probability of certain cardiometabolic risks, including a high BMI and high non-HDL cholesterol ${ }^{(45)}$. There are clear opportunities to better align agricultural policies with public health outcomes by reducing or eliminating 
subsidies on beef and milk and increasing the subsidies on fruit and vegetables ${ }^{(43,44)}$. This also shows that food prices are indeed a policy concern (not only that of the free market) where we rely on solid food policy initiatives (covering health, environmental sustainability and the economy) to ensure a nutritious food supply now and for future generations.

Wilma E Waterlander National Institute for Health Innovation School of Population Health University of Auckland

Auckland, New Zealand Email: w.waterlander@auckland.ac.nz

Sally Mackay Department of Epidemiology and Biostatistics School of Population Health University of Auckland Auckland, New Zealand

\section{References}

1. Colchero MA, Popkin BM, Rivera JA et al. (2016) Beverage purchases from stores in Mexico under the excise tax on sugar sweetened beverages: observational study. BMJ 352, h6704.

2. Darmon N \& Drewnowski A (2015) Contribution of food prices and diet cost to socioeconomic disparities in diet quality and health: a systematic review and analysis. Nutr Rev 73, 643-660.

3. Waterlander WE, de Mul A, Schuit AJ et al. (2010) Perceptions on the use of pricing strategies to stimulate healthy eating among residents of deprived neighbourhoods: a focus group study. Int J Behav Nutr Phys Act 7, 44.

4. Darmon N, Ferguson EL \& Briend A (2006) Impact of a cost constraint on nutritionally adequate food choices for French women: an analysis by linear programming. J Nutr Educ Behav 38, 82-90.

5. Rao M, Afshin A, Singh G et al. (2013) Do healthier foods and diet patterns cost more than less healthy options? A systematic review and meta-analysis. BMJ Open 3, 12 .

6. Lewis M \& Lee A (2016) Costing 'healthy' food baskets in Australia: a systematic review of food price and affordability monitoring tools, protocols and methods. Public Health Nutr 19, 2872-2886.

7. Swinburn B, Sacks G, Vandevijvere S et al. (2013) INFORMAS (International Network for Food and Obesity/ non-communicable diseases Research, Monitoring and Action Support): overview and key principles. Obes Rev 14, Suppl., 1, 1-12.

8. Lee AJ, Kane S, Ramsey R et al. (2016) Testing the price and affordability of healthy and current (unhealthy) diets and the potential impacts of policy change in Australia. BMC Public Health 16, 315.

9. US Department of Health and Human Services \& US Department of Agriculture (2005) Dietary Guideline for Americans, 2005, 7th ed. Washington, DC: US Government Printing Office.

10. Drewnowski A (2003) Fat and sugar: an economic analysis. J Nutr 133, issue 3, 8385-840S.

11. Drewnowski A (2004) Obesity and the food environment: dietary energy density and diet costs. Am J Prev Med 27, 3 Suppl., 154-162.
12. Darmon N, Briend A \& Drewnowski A (2004) Energy-dense diets are associated with lower diet costs: a community study of French adults. Public Health Nutr 7, 21-27.

13. Drewnowski A, Darmon N \& Briend A (2004) Replacing fats and sweets with vegetables and fruits - a question of cost. Am J Public Health 94, 1555-1559.

14. Drewnowski A, Monsivais P, Maillot M et al. (2007) Lowenergy-density diets are associated with higher diet quality and higher diet costs in French adults. J Am Diet Assoc 107, 1028-1032.

15. Lipsky LM (2009) Are energy-dense foods really cheaper? Reexamining the relation between food price and energy density. Am J Clin Nutr 90, 1397-1401.

16. Darmon N \& Maillot M (2010) In foods, energy is cheap where it is abundant and expensive where it is scarce: this is a fact, not an artifact. Am J Clin Nutr 91, 1068-1069.

17. Carlson A \& Frazao E (2014) Food costs, diet quality and energy balance in the United States. Physiol Behav 134, 20-31.

18. Temple NJ \& Steyn NP (2009) Food prices and energy density as barriers to healthy food patterns in Cape Town, South Africa. J Hunger Environ Nutr 4, 203-213.

19. Drewnowski A (2015) Nutrition economics: how to eat better for less. J Nutr Sci Vitaminol (Tokyo) 61, Suppl., S69-S71.

20. Marty L, Dubois C, Gaubard MS et al. (2015) Higher nutritional quality at no additional cost among low-income households: insights from food purchases of 'positive deviants'. Am J Clin Nutr 102, 190-198.

21. Drewnowski A (2010) The Nutrient Rich Foods Index helps to identify healthy, affordable foods. Am J Clin Nutr 91, issue 4, 1095S-1101S.

22. Maillot M, Ferguson EL, Drewnowski A et al. (2008) Nutrient profiling can help identify foods of good nutritional quality for their price: a validation study with linear programming. J Nutr 138, 1107-1113.

23. Primavesi L, Caccavelli G, Ciliberto A et al. (2015) Nutrieconomic model can facilitate healthy and low-cost food choices. Public Health Nutr 18, 827-835.

24. Glanz K, Basil M, Maibach E et al. (1998) Why Americans eat what they do: taste, nutrition, cost, convenience, and weight control concerns as influences on food consumption. J Am Diet Assoc 98, 1118-1126.

25. McDermott AJ \& Stephens MB (2010) Cost of eating: whole foods versus convenience foods in a low-income model. Fam Med 42, 280-284.

26. Celnik D, Gillespie L \& Lean MEJ (2012) Time-scarcity, ready-meals, ill-health and the obesity epidemic. Trends Food Sci Technol 27, 4-11.

27. Luiten CM, Steenhuis IH, Eyles H et al. (2016) Ultraprocessed foods have the worst nutrient profile, yet they are the most available packaged products in a sample of New Zealand supermarkets. Public Health Nutr 19, 530-538.

28. Stuckler D, McKee M, Ebrahim S et al. (2012) Manufacturing epidemics: the role of global producers in increased consumption of unhealthy commodities including processed foods, alcohol, and tobacco. PLoS Med 9, 6 .

29. Moodie R, Stuckler D, Monteiro C et al. (2013) Profits and pandemics: prevention of harmful effects of tobacco, alcohol, and ultra-processed food and drink industries. Lancet 381, 670-679.

30. Monteiro CA, Levy RB, Claro RM et al. (2010) A new classification of foods based on the extent and purpose of their processing. Cad Saude Publica 26, 2039-2049.

31. Pan American Health Organization \& World Health Organization (2015) Ultra-Processed Food and Drink Products in Latin America: Trends, Impact on Obesity, Policy Implications. Washington, DC: PAHO/WHO.

32. Mancino L \& Newman C (2007) Who Has Time to Cook? How Family Resources Influence Food Preparation, 
Economic Research Report no. ERR-40]. Washington, DC: US Department of Agriculture, Economic Research Service.

33. Davis GC \& You W (2011) Not enough money or not enough time to satisfy the Thrifty Food Plan? A cost difference approach for estimating a money-time threshold. Food Policy 36, 101-107.

34. Yang Y, Davis GC \& Muth MK (2015) Beyond the sticker price: including and excluding time in comparing food prices. Am J Clin Nutr 102, 165-171.

35. An R (2013) Effectiveness of subsidies in promoting healthy food purchases and consumption: a review of field experiments. Public Health Nutr 16, 1215-1228.

36. Epstein LH, Jankowiak N, Nederkoorn C et al. (2012) Experimental research on the relation between food price changes and food-purchasing patterns: a targeted review. Am J Clin Nutr 95, 789-809.

37. Eyles H, Ni Mhurchu C, Nghiem N et al. (2012) Food pricing strategies, population diets, and non-communicable disease: a systematic review of simulation studies. PLoS Med $\mathbf{9}$, e1001353.

38. Finkelstein EA, Strombotne KL, Zhen C et al. (2014) Food prices and obesity: a review. Adv Nutr 5, 818-821.

39. Powell LM, Chriqui JF, Khan T et al. (2013) Assessing the potential effectiveness of food and beverage taxes and subsidies for improving public health: a systematic review of prices, demand and body weight outcomes. Obes Rev $\mathbf{1 4}$, $110-128$.

40. Thow AM, Downs S \& Jan S (2014) A systematic review of the effectiveness of food taxes and subsidies to improve diets: understanding the recent evidence. Nutr Rev $\mathbf{7 2}$, 551-565.

41. Briggs AD, Kehlbacher A, Tiffin R et al. (2016) Simulating the impact on health of internalising the cost of carbon in food prices combined with a tax on sugar-sweetened beverages. BMC Public Health 16, 107.

42. Foley JA, Ramankutty N, Brauman KA et al. (2011) Solutions for a cultivated planet. Nature 478, 337-342.

43. Birt C (2007) A CAP on Health? The Impact of the EU Common Agricultural Policy on Public Health. London: Faculty of Public Health.

44. Popkin BM (2009) Reducing meat consumption has multiple benefits for the world's health. Arch Intern Med 169, 543-545.

45. Siegel KR, McKeever Bullard K, Imperatore G et al. (2016) Association of higher consumption of foods derived from subsidized commodities with adverse cardiometabolic risk among US adults. JAMA Intern Med 176, 1124-1132.

46. Waterlander WE, de Haas WE, van Amstel I et al. (2010) Energy density, energy costs and income - how are they related? Public Health Nutr 13, 1599-1608. 